\title{
Operando SAXS Study of a Pt/C Fuel Cell Catalyst with an X-ray Laboratory
} Source

Johanna Schröder ${ }^{1+}$, Jonathan Quinson ${ }^{2+^{*}}$, Jacob J. K. Kirkensgaard ${ }^{3,4}$, and Matthias Arenz ${ }^{2^{*}}$

${ }^{1}$ Department of Chemistry and Biochemistry University of Bern, Bern, Switzerland

${ }^{2}$ Department of Chemistry, University of Copenhagen, Copenhagen, Denmark

${ }^{3}$ Department of Food Science, University of Copenhagen, Frederiksberg, Denmark

${ }^{4}$ Niels Bohr Institute, University of Copenhagen, Copenhagen, Denmark

+ equally contributing authors

*corresponding authors, e-mails: jonathan.quinson@chem.ku.dk; matthias.arenz@dcb.unibe.ch

\begin{abstract}
Small angle X-ray scattering (SAXS) is a powerful technique to investigate the degradation of catalyst materials. Ideally such investigations are performed operando, i.e., during a catalytic reaction. An example of operando measurements is to observe the degradation of fuel cell catalysts during an accelerated stress test (AST). Fuel cell catalysts consist of Pt or Pt alloy nanoparticles (NPs) supported on a high surface area carbon. A key challenge of operando SAXS measurements is a proper background subtraction of the carbon support to extract the information of the size distribution of the Pt NPs as a function of the AST treatment. Typically, such operando studies require the use of synchrotron facilities. The background measurement can then be performed by anomalous SAXS (aSAXS) or in a grazing incidence configuration. In this work we present a proof-of-concept study demonstrating the use of a laboratory X-ray diffractometer for operando SAXS. Data acquisition of operando SAXS with a laboratory Xray diffractometer is desirable due to the general challenging and limited accessibility of synchrotron facilities. They become even more crucial under the ongoing and foreseen restrictions related to the COVID-19 pandemic. Although, it is not the aim to completely replace synchrotron-based studies, it is shown that the background subtraction can be achieved by a simple experimental consideration in the setup that can ultimately facilitate operando SAXS measurements at a synchrotron facility.
\end{abstract}

Keywords: operando spectroscopy, small angle X-ray scattering, fuel cell catalyst, degradation

\section{Introduction}

Proton exchange membrane fuel cells (PEMFCs) are seen as an important renewable alternative of combustion engines, especially for large vehicles and heavy duty trucks. [1-4] For an efficient conversion of the hydrogen fuel to electric power, highly active catalysts are required. PEMFC catalysts are often composed of Pt or Pt alloy nanoparticles (NPs). [5,6] Besides a high catalytic activity, efficient catalysts also require sufficient stability. [2,7] The stability of catalysts is often investigated by performing accelerated stress tests (ASTs) to decrease the testing time. [8,9] Such ASTs were recently performed on commercial Pt catalysts in gas diffusion electrode (GDE) setups [10-12] simulating load cy- 
cles and start-stop conditions as recommended by the Fuel Cell Commercialization Conference of Japan (FCCJ) $[13,14]$ under realistic mass transport conditions. To determine changes in particle size as result of the AST protocol the measurements in the GDE setup were coupled to ex situ small-angle X-ray scattering (SAXS). [11,12] However, ex situ measurements provide only the possibility to determine the particle size at few points in time (i.e., at the beginning and the end of treatment). To establish a timeresolved picture of catalyst degradation the number of samples would need to be enormously increased.

To obtain a deeper insight into time-resolved phenomena, in situ [15-17] or operando [18-20] SAXS is necessary, which typically is performed at synchrotron X-ray sources. Beamtime for operando SAXS measurements at a synchrotron facility to investigate time-resolved degradation mechanisms is unfortunately limited, challenging to get granted, and typically requires a long time until the proposals are accepted, and the measurements can be performed. Furthermore, planning a beamtime at a synchrotron can be costly and logistically challenging. For instance, equipment must be shipped to the synchrotron facility, making the measurements rather inflexible already under normal circumstances. The COVID-19 pandemic made planned travelling basically impossible in 2020 and currently restricts most measurements at synchrotrons to remote operation which is even more challenging. Being able to study the time resolved degradation of catalysts with operando SAXS using a laboratory X-ray source would not be only at the moment but also for the future very helpful; it is resource-efficient to reduce travelling and research costs. Additionally, the use of a laboratory source gives the possibility for better designed experiments, e.g., more and more reliable repeats. At a synchrotron repeating measurements and checking the reproducibility of measurements is often limited due to the scarce beamtime. It can take months before experiments can be repeated.

In this work an operando SAXS and X-ray absorption spectroscopy (XAS) cell was used with some adjustments to the firstly introduced version by Binninger et al. [18] for measurements at synchrotron Xray sources. This operando cell was used in this study in a laboratory X-ray source to perform electrochemical ASTs and investigate the time resolved degradation of a Pt NP catalyst with two distinguishable size populations by SAXS. An important point to obtain reasonable data for the particle size probability densities retrieved from SAXS measurements is a proper background subtraction, implying a careful background measurement. Using laboratory X-ray sources, the background measurement usually requires a separate experiment leading often to difficulties in normalization of the curves. [21] At synchrotron facilities anomalous SAXS can be performed by tuning the X-ray energy to subtract elemental scattering energies. [21] Alternatively the measurements can be performed in grazing incidence. [22-24] These two choices, however, are not available for measurements at laboratory X-ray sources. Tillier et al. [19] thus measured the background ex situ on a Pt-free carbon electrode performing the same degradation protocol as on the sample which led to the measurement of a suitable background.

Here we present a new design of the working electrode in the operando cell. In this study, the catalyst film as well as a catalyst-free part of the substrate (i.e., the background) experience the same electrochemical treatment during the AST, enabling an operando SAXS background measurement. It is demonstrated that with such a design operando studies of catalyst degradation can be performed even with less powerful X-ray sources. The same technique can also be used to facilitate operando SAXS measurements at a synchrotron facility. 


\section{Experimental}

\subsection{Chemicals and materials}

For the catalyst ink formation and the cleaning of the cell ultrapure Milli-Q water (resistivity > 18.2 $\mathrm{M} \Omega \cdot \mathrm{cm}$, total organic carbon (TOC) $<5 \mathrm{ppb}$ ) from a Millipore system was used. The catalysts ink was prepared with isopropanol (IPA, 99.7+\%, Alfa Aesar), commercial Pt/C catalysts (TEC10E20A (1-2 nm $\mathrm{Pt} / \mathrm{C}, 19.4 \mathrm{wt} \% \mathrm{Pt}$ ) and TEC10E50E-HT (4-5 nm Pt/C, $50.6 \mathrm{wt} \% \mathrm{Pt})$, Tanaka kikinzoku kogyo), and Nafion dispersion (D1021, 10 wt. \%, EW 1100, Fuel Cell Store). The working electrolyte (WE) was prepared by a gas diffusion layers (GDL) with a microporous layer (MPL) on top (Freudenberg H23C8, $0.230 \mu \mathrm{m}$ thick) and a leather punching tool (Takagi Co., 4907052141636, diameter of $3 \mathrm{~mm}$ ) was used to cut the GDL to the desired size. In the electrochemical measurements $0.1 \mathrm{M} \mathrm{HClO}_{4}$ prepared from 70 $\%$ perchloric acid $\left(\mathrm{HClO}_{4}\right.$, Suprapur®, Merck) was used as electrolyte.

\subsection{Catalyst synthesis}

The catalyst mixture with two size populations on different carbon flakes was obtained by mixing $10.31 \mathrm{mg}$ TEC10E20A and $5.97 \mathrm{mg}$ TEC10E50E-HT (Pt mass ratio of 40:60 (small:large NPs)). The ink with a Pt concentration of $0.5 \mathrm{mg} \mathrm{mL}^{-1}$ was formed by dispersing the catalysts in $10 \mathrm{~mL}$ of a mixture of Milli-Q water and IPA (water/IPA ratio of 3:1) as described before [11]. The mixture was sonicated for $10 \mathrm{~min}$ in a sonication bath to get a good dispersion. $53 \mu \mathrm{L}$ of Nafion was added (Nafion/carbon mass ratio of 1). The dispersion was again sonicated for $10 \mathrm{~min}$ in a sonication bath.

\subsection{Catalyst film preparation}

The Freudenberg GDL was placed in a vacuum filtration setup between a glass funnel and a sand core filter. All this was placed on a collecting bottle as described by Yarlagadda et al. [25] $4 \mathrm{~mL}$ of the inks were diluted with $7 \mathrm{~mL}$ of Milli-Q water and $29 \mathrm{~mL}$ of IPA (water-IPA ratio of 1:3, Pt concentration of $\left.0.05 \mathrm{mg} \mathrm{L}^{-1}\right)$. The mixture was sonicated for $1 \mathrm{~min}$. The diluted ink was then filled in a funnel. A diaphragm vacuum pump (Vacuubrand, $\mathrm{MZ} 2 \mathrm{C}$, max. $1.7 \mathrm{~m}^{3} / \mathrm{h}$ and $9.0 \mathrm{mbar}$ ) was used to deposit the catalyst on top of the GDL (duration for the filtering was ca. $4 \mathrm{~h}$ ). Afterwards, the GDE was dried and stored in air. By this procedure a theoretical Pt loading of $0.208 \mathrm{mg} \mathrm{cm}_{\text {geo }}^{-2}$, typically used for commercial fuel cells [26] is obtained.

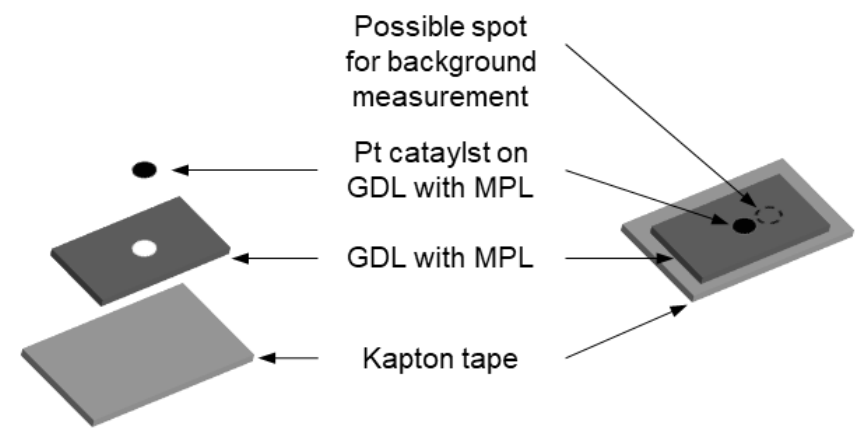

Figure 1. Sketch of the WE (diameter of $3 \mathrm{~mm}$ ) consist of the Pt catalyst on a GDL with a MPL placed in the middle of a GDL and glued to Kapton tape. 


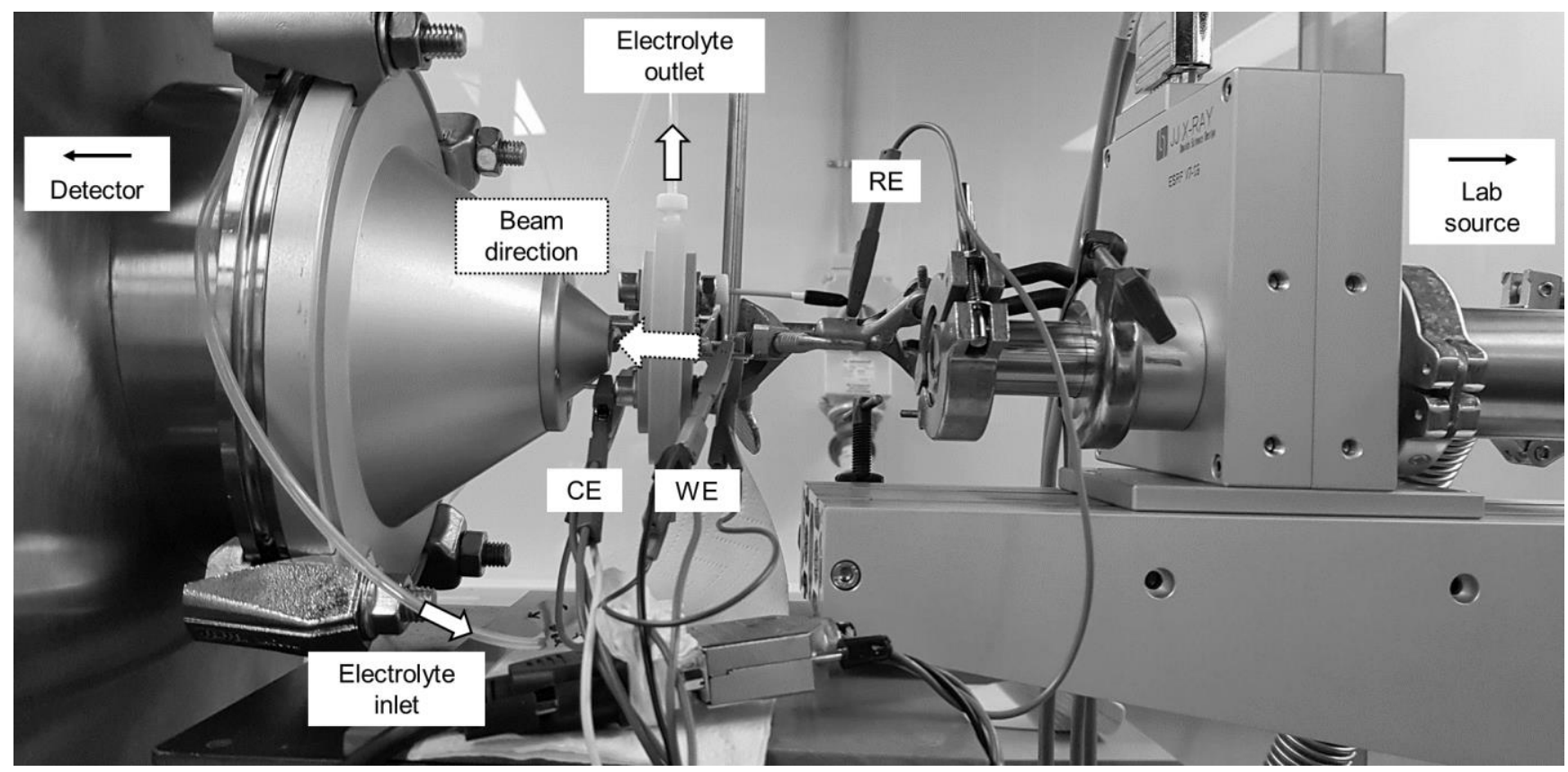

Figure 2. Experimental setup of the operando cell placed in the beam made by the SAXS lab source.

\subsection{Degradation study in operando cell}

The cell used for operando SAXS is adapted from the one reported by Binninger et al. [18] As counter electrode the Freudenberg GDL was used and as reference electrode (RE) an Ag wire. To prepare the working electrode (WE) a circle (diameter of $3 \mathrm{~mm}$ ) was punched inside a rectangle of the Freudenberg GDL. This rectangle was fixed on a Kapton tape (MPL was placed upside). A circle (diameter of $3 \mathrm{~mm}$ ) of the catalyst film deposited on the GDL by vacuum filtration was placed in the hole, see Figure 1 . The electrolyte $\left(0.1 \mathrm{M} \mathrm{HClO}_{4}\right)$ exposed to air was manually pulled through the cell before and after an electrochemical measurement by drawing up a $50 \mathrm{~mL}$ syringe. For the AST measurement the potential was stepped between 0.6 and $1.0 \mathrm{~V}_{\text {RHE }}$ with $3 \mathrm{~s}$ holding time at each potential. The applied potential was adjusted to the open circuit potential of the cell: $0.422 \mathrm{~V}$ (steps between 0.12 and $\left.0.52 \mathrm{~V}_{\mathrm{Ag}}\right), 0.415 \mathrm{~V}(0.11$ and $\left.0.51 \mathrm{~V}_{\mathrm{Ag}}\right), 0.406 \mathrm{~V}\left(0.10\right.$ and $\left.0.50 \mathrm{~V}_{\mathrm{Ag}}\right), 0.394 \mathrm{~V}\left(0.09\right.$ and $\left.0.49 \mathrm{~V}_{\mathrm{Ag}}\right)$, and $0.406(0.10$ and 0.50 $\left.\mathrm{V}_{\mathrm{Ag}}\right)$. The resistance during the measurement was determined online using an AC signal $\left(5 \mathrm{kHz}, 5 \mathrm{x} 10^{-5}\right.$ A).[27] Details about the AST protocol and data are found in the SI, see Figure S1 and S2.

\subsection{Small-angle X-ray Scattering (SAXS)}

The size change of NPs supported on carbon was assessed by SAXS measurements as previously reported. [11,12] SAXS measurements were performed at the Niels Bohr Institute, University of Copenhagen, Denmark, on a Ganesha instrument (SAXSLab). The Ganesha is equipped with a 100XL+ microfocus sealed X-ray tube (Rigaku), producing a photon beam with a wavelength of $1.54 \AA$, and a 2D 300 $\mathrm{K}$ Pilatus detector (Dectris). The detector is situated inside a large continuous vacuum chamber, where it can be moved to adjust the distance between sample and detector. For the present experiment, the sample detector distance was set to cover a q-range of 0.0159-0.7428 $\AA^{-1}$ calibrated using silver behenate. Motorized scatterless slits allow to tune the collimation and for the present study a 2-slit configuration was used with $0.9 \mathrm{~mm}$ and $0.4 \mathrm{~mm}$ squares as first and second pinholes, respectively, resulting in a beam 
with ca. $10 \mathrm{M}$ photons/second hitting the sample. The two-dimensional scattering data were azimuthally averaged, normalized by the incident radiation intensity, the sample exposure time, and the transmission. Using a standard SAXSGUI reduction software, the data were then corrected for the background and detector inhomogeneities. The samples consisting of the NPs on the GDL were placed in the operando cell and the cell exposed to the beam produced by the lab source. The cell itself was not under vacuum, but the detector was placed in a chamber under vacuum (on the left of the image in Figure 2). The background was measured next to the Pt catalyst (see Figure 1) by manually moving the operando cell. A fast SAXS measurement of $15 \mathrm{~s}$ was performed to confirm that a metal-free spot was subjected to the beam.

The data analysis after background subtraction (acquired in a part of the sample without Pt NPs) was performed as described previously $[11,12,28,29]$ using a polydisperse spheres model. The radially averaged intensity $\mathrm{I}(\mathrm{q})$ is expressed as a function of the scattering vector $\mathrm{q}=4 \pi \cdot \sin (\theta) / \lambda$, where $\lambda$ is the wavelength, and $2 \theta$ is the scattering angle. The background-corrected scattering data were fitted using a power law to consider the behavior at low q value and a model of one or two polydisperse spheres described by a volume-weighted log-normal distribution. The scattering data are fitted to the following expression:

$$
\mathrm{I}(\mathrm{q})=\mathrm{A} \cdot \mathrm{q}^{-\mathrm{n}}+\mathrm{C}_{1} \cdot \int \mathrm{P}_{\mathrm{s} 1}(\mathrm{q}, \mathrm{R}) \mathrm{V}_{1}(\mathrm{R}) \mathrm{D}_{1}(\mathrm{R}) \mathrm{dR}+\mathrm{C}_{2} \cdot \int \mathrm{P}_{\mathrm{s} 2}(\mathrm{q}, \mathrm{R}) \mathrm{V}_{2}(\mathrm{R}) \mathrm{D}_{2}(\mathrm{R}) \mathrm{dR}
$$

where $A \cdot q^{-n}$ corresponds to the power law with $A$ and $n$ as free parameters, $C_{1}$ and $C_{2}$ are scaling constants, $\mathrm{P}_{\mathrm{s} 1}$ and $\mathrm{P}_{\mathrm{s} 2}$ the sphere form factors, $\mathrm{V}_{1}$ and $\mathrm{V}_{2}$ the particle volumes and $\mathrm{D}_{1}$ and $\mathrm{D}_{2}$ the $\log$-normal size distribution. The sphere form factor is given by: [30,31]

$$
\mathrm{P}_{\mathrm{s}}(\mathrm{q}, \mathrm{R})=\left(3 \frac{\sin (\mathrm{qR})-\mathrm{qR} \cos (\mathrm{qR})}{(\mathrm{qR})^{3}}\right)^{2}
$$

and the log-normal distribution by:

$$
\mathrm{D}(\mathrm{R})=\frac{1}{\mathrm{R} \sigma \sqrt{2 \pi}} \exp \left(\frac{-\left[\ln \left(\frac{\mathrm{R}}{\mathrm{R}_{0}}\right)\right]^{2}}{2 \sigma^{2}}\right)
$$

where $\sigma$ is the variance and $\mathrm{R}_{0}$ (evaluated in $\AA$ ) the geometric mean of the log-normal distribution. The fitting was done using a home written MATLAB code. The free parameters in the model are A, n, $\mathrm{C}_{1}, \mathrm{R}_{1}, \sigma_{1}, \mathrm{C}_{2}, \mathrm{R}_{2}, \sigma_{2}$. The values obtained for these parameters are reported in Table $\mathrm{S} 1$. In order to account for the two populations, the reported probability density functions were weighted by the relative surface contribution of the spheres as detailed in the SI. The scattering data and corresponding fits are reported in Figures S3-S6.

The scattering intensities were recorded for 15 minutes per measurement, with and without electrolyte in the operando cell, before the AST and after 150, 300, 450, 600, and 900 steps of electrochemical treatments, as detailed below and indicated in the different Figures.

\section{Results and discussion}

As discussed in the introduction, establishing a proper background using an laboratory X-ray source for SAXS is often difficult due to the need for separate (ex situ) measurements of the background and sample scattering data. [21] In this study, we demonstrate a simple design that enables to record the background data by moving the cell slightly horizontally to switch between the beam spot hitting the 
catalysts or the catalyst-free GDL next to the catalyst sample (i.e., operando), respectively. With the help of the specific WE configuration, see also the experimental part and Figure 1, the catalyst and the "blank" WE on which the background data are recorded, receive the same electrochemical treatment.

To prove the concept, two commercial Pt NP catalysts supported on carbon (Pt/C) consisting of two distinguishable size populations were mixed together. This mixed catalyst powder enables to investigate the influence of background subtraction over a range of particle sizes. Additionally, particle size dependent dissolution rates [32,33] or degradation mechanism [34] were reported before. Due to the two size populations the degradation mechanism of Ostwald ripening is expected to be favored, i.e., that the small particles grow at the expense of large ones. [35]

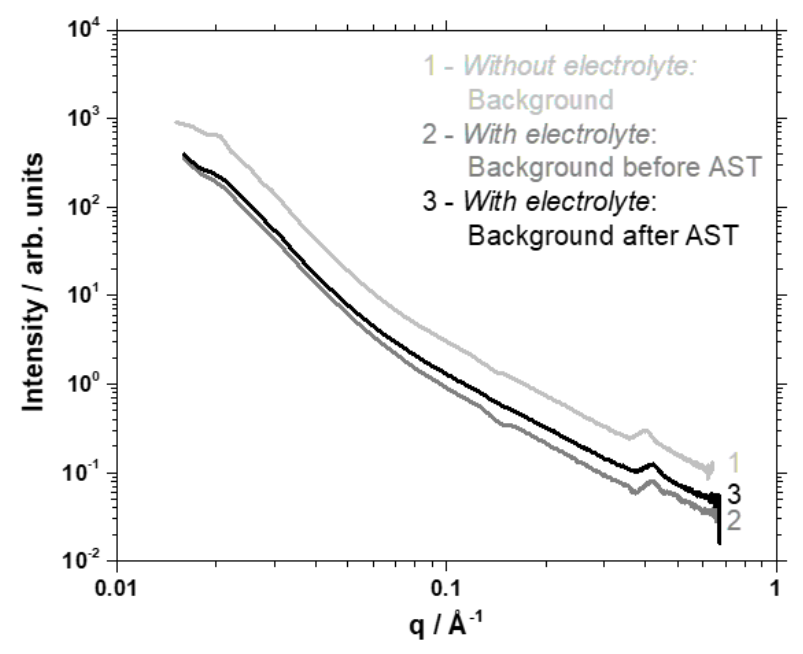

Figure 3. Overview of signal intensity for the SAXS measurements for backgrounds acquired under different conditions as indicated.

Figure 3 compares different background scattering data that were measured in the described assembly before subjecting the WE to the electrolyte (light grey line), after introducing electrolyte to the SAXS cell (grey line), and after the electrochemical AST study (black line). After introducing the electrolyte to the operando cell an AST protocol with potential steps consisting of $3 \mathrm{~s}$ holds at 0.6 and $1 \mathrm{~V}_{\mathrm{RHE}}$, respectively, was applied, see Figure S1 and S2, to observe the degradation of the catalyst film with the Pt/C consisting of two size populations. The difference in the obtained probability density function for the size distributions of the $\mathrm{Pt} / \mathrm{C}$ catalysts is demonstrated in Figure 4. The retrieved probability density functions are shown for using background data collected at the blank GDL exposed to electrolyte, but before applying the AST (Figure 3a) as well as when using background data collected after exposing the blank GDL to the same electrochemical treatment as the sample (Figure 3b). In addition, the probability density functions for the pristine ex situ Pt/C sample are shown in both Figures.

It is directly apparent that recording the background data before applying the AST as done in Figure 3 is not optimal. Although one can distinguish the two size populations of the mixed catalyst, see Figure 4, and follow the change in the populations, there is a clear difference between the size probability density of the pristine ex situ sample (measured without electrolyte) and the one before applying the electrochemical AST treatment (measured with electrolyte). This effect is pronounced for the population of the 
smaller Pt NPs and might have several origins: It could be that the electrolyte was not in a steady state within the cell or that the metal-free spot chosen for the background measurement was not adequately selected. However, the likely explanation is that since the intensity scales with the square of the volume, small NPs are more sensitive to the added scattering and absorption from the electrolyte. It then becomes easier to account for the 'larger NPs' in presence of electrolyte. This leads to probability densities where the larger NPs account for more of the size distribution than the smaller ones, see Figure 4. Nevertheless, it also leads to an erroneous estimation of the average particle size of the smaller Pt NPs, see Table S1. In addition, no strong difference in the average particle size of the small NPs is seen during the complete AST; after 600 potential steps only the size distribution widens, see Figure 4 and Table S1. This trend is not expected based on previous investigations where at least some changes in the average particle size are reported. [11]

a)

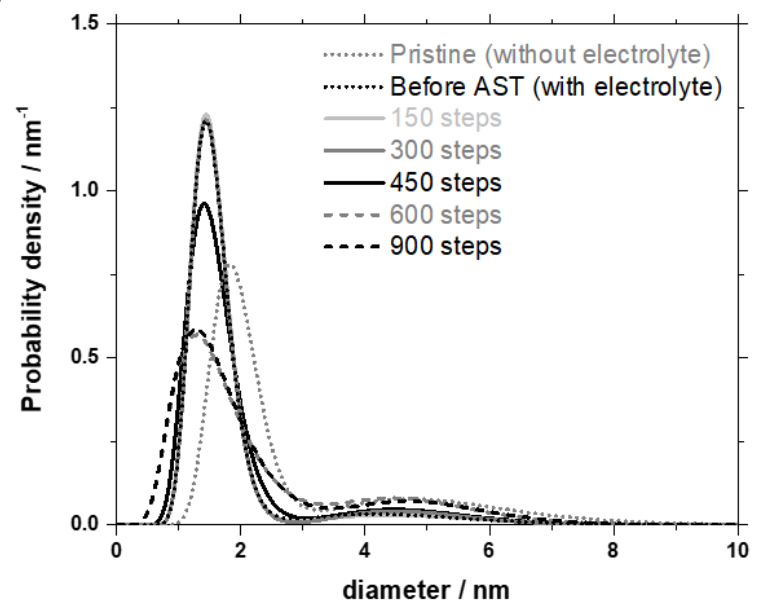

b)

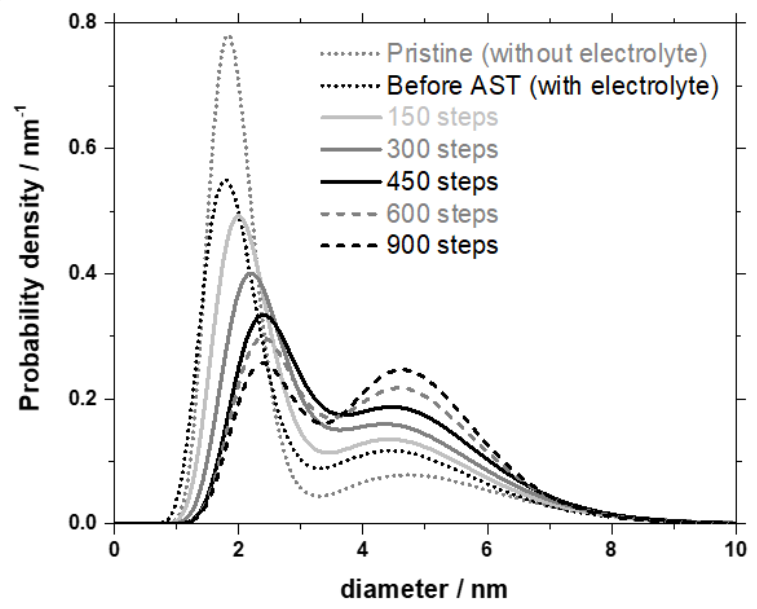

Figure 4. Probability density function retrieved from SAXS data analysis using as background scattering data measured a) before and b) after the electrochemical test.

By comparison, using background data of the GDL that experienced the same electrochemical treatment as the catalyst, the mean particle size of the two size populations of the pristine sample (without electrolyte) are as expected at the same size position as for the set of data measured before the AST but with electrolyte, see Figure 4 and Figure S7. Nevertheless, also with a proper background subtraction, a difference between the pristine sample and the one in presence of the electrolyte is observed. In particular a decrease of the probability density of the population with small particle size at the expense of the one with larger size is seen. This observation could be an indication for particle dissolution followed by Ostwald ripening. Indeed, it is observed in scanning flow cell (SFC) measurements coupled to inductively coupled plasma mass spectrometry (ICP-MS) that upon the contact of a Pt/C catalyst with an electrolyte $\mathrm{Pt}$ dissolution is induced. [8,36] Applying the AST, it is observed that the size population of the small particles increases from $2.0 \pm 0.5 \mathrm{~nm}$ to $2.6 \pm 0.6 \mathrm{~nm}$, while the large particles remain constant at $5.0 \pm 1.4$ before and $5.0 \pm 1.1 \mathrm{~nm}$ after 900 steps. This behavior of particle growth of small particles (1-2 $\mathrm{nm})$ and stable larger NPs $(4-5 \mathrm{~nm})$ is consistent with results observed recently in a GDE setup investigating both catalysts independently by ex situ SAXS. [11] It could not be distinguished between the deg- 
radation mechanisms of particle dissolution with subsequent Ostwald ripening or particle coalescence; both mechanisms were in agreement with the observed NP growth. Therefore, the comparison of the initial and end of treatment size populations between ex situ (GDE setup [11]) and in situ SAXS (operando cell) seem to show that the presence of the second size population in the catalyst mixture in the operan$d o$ cell does not have an influence on the degradation mechanism. However, the catalyst mixture in the operando SAXS result in a continuous size increase and reduced probability density of the small population, while the probability density of the large particles increases at the same time. Consequently, operando SAXS points toward a continuous process of Pt dissolution with subsequent Ostwald ripening as compared to particle coalescence.

\section{Conclusion}

It is demonstrated how using a SAXS laboratory X-ray source not only enables a proper background subtraction, but also high-quality degradation data can be obtained. Our approach has a strong benefit because beamtimes and the flexibility of the experiments at synchrotron X-ray sources are limited. There is often too little room for optimization of the experimental design during synchrotron beamtime to implement spontaneous ideas and to repeat experiments under optimized conditions can take quite a long time. In addition, the synchrotron beamtime itself includes travelling and the transport of materials to the synchrotron beamline which can be a large cost factor for research groups and is virtually impossible during the current COVID-19 pandemic. In contrast, experiments using a laboratory X-ray source can be performed on-site at universities and repeats of experiments can be more easily planned. Last but not least, on-site experiments offer a great opportunity to perform preliminary studies to optimize chances to secure synchrotron beamtimes by improving beamtime proposals as well as to make the most of the valuable time at synchrotrons if the proposal is granted.

\section{Acknowledgements}

This work was supported by the Swiss National Science Foundation (SNSF) via the project No. 200021_184742 and the Danish National Research Foundation Center for High Entropy Alloys Catalysis (CHEAC) DNRF-149.

\section{References}

[1] Gröger O, Gasteiger H A and Suchsland J P 2015 Review-Electromobility: Batteries or fuel cells? J. Electrochem. Soc. 162 A2605-22

[2] Stacy J, Regmi Y N, Leonard B and Fan M 2017 The recent progress and future of oxygen reduction reaction catalysis: A review Renew. Sustain. Energy Rev. 69 401-14

[3] Nazir H, Muthuswamy N, Louis C, Jose S, Prakash J, Buan M E M, Flox C, Chavan S, Shi X, Kauranen P, Kallio T, Maia G, Tammeveski K, Lymperopoulos N, Carcadea E, Veziroglu E, Iranzo A and M. Kannan A 2020 Is the H2 economy realizable in the foreseeable future? Part III: $\mathrm{H} 2$ usage technologies, applications, and challenges and opportunities Int. J. Hydrogen Energy 45 28217-39 
[4] Ruf Y, Baum M, Zorn T, Menzel A and Rehberger J 2020 Study Report: Fuel Cells Hydrogen Trucks - Heavy-Duty's High Performance Green Solution Study Summary, published 15 Dec 2020 by Fuel Cells and Hydrogen Joint Undertaking (FCH JU) and Roland Berger, https://www.fch.europa.eu/publications/study-fuel-cells-hydrogen-trucks (accessed last 24 Mar 2021)

[5] Yoshida T and Kojima K 2015 Toyota MIRAI Fuel Cell Vehicle and Progress Toward a Future Hydrogen Society Electrochem. Soc. Interface 24 45-9

[6] Pollet B G, Kocha S S and Staffell I 2019 Current status of automotive fuel cells for sustainable transport Curr. Opin. Electrochem. 16 90-5

[7] Katsounaros I, Cherevko S, Zeradjanin A R and Mayrhofer K J J J 2014 Oxygen electrochemistry as a cornerstone for sustainable energy conversion Angew. Chemie - Int. Ed. 53 $102-21$

[8] Pizzutilo E, Geiger S, Grote J-P, Mingers A, Mayrhofer K J J, Arenz M and Cherevko S 2016 On the Need of Improved Accelerated Degradation Protocols (ADPs): Examination of Platinum Dissolution and Carbon Corrosion in Half-Cell Tests J. Electrochem. Soc. 163 F1510-4

[9] Yano H, Watanabe M, Iiyama A and Uchida H 2016 Particle-size effect of Pt cathode catalysts on durability in fuel cells Nano Energy 29 323-33

[10] Alinejad S, Inaba M, Schröder J, Du J, Quinson J, Zana A and Arenz M 2020 Testing fuel cell catalysts under more realistic reaction conditions: accelerated stress tests in a gas diffusion electrode setup J. Phys. Energy 2024003

[11] Schröder J, Quinson J, Mathiesen J K, Kirkensgaard J J K, Alinejad S, Mints V A, Jensen K M Ø and Arenz M 2020 A New Approach to Probe the Degradation of Fuel Cell Catalysts under Realistic Conditions: Combining Tests in a Gas Diffusion Electrode Setup with Small Angle Xray Scattering J. Electrochem. Soc. 167134515

[12] Alinejad S, Quinson J, Schröder J, Kirkensgaard J J K and Arenz M 2020 Carbon-supported platinum electrocatalysts probed in a gas diffusion setup with alkaline environment: How particle size and mesoscopic environment influence the degradation mechanism ACS Catal. 10 13040-9

[13] Ohma A, Shinohara K, Iiyama A, Yoshida T and Daimaru A 2019 Membrane and Catalyst Performance Targets for Automotive Fuel Cells by FCCJ Membrane, Catalyst, MEA WG ECS Trans. 41 775-84

[14] Park Y-C, Kakinuma K, Uchida M, Tryk D A, Kamino T, Uchida H and Watanabe M 2013 Investigation of the corrosion of carbon supports in polymer electrolyte fuel cells using simulated start-up/shutdown cycling Electrochim. Acta 91 195-207

[15] Harada M, Tamura N and Takenaka M 2011 Nucleation and growth of metal nanoparticles during photoreduction using in situ time-resolved SAXS analysis J. Phys. Chem. C 115 14081-92

[16] Wu L, Willis J J, McKay I S, Diroll B T, Qin J, Cargnello M and Tassone C J 2017 Hightemperature crystallization of nanocrystals into three-dimensional superlattices Nature $\mathbf{5 4 8} 197$ 201

[17] Quinson J, Neumann S, Kacenauskaite L, Bucher J, Kirkensgaard J J K, Simonsen S B, Theil Kuhn L, Zana A, Vosch T, Oezaslan M, Kunz S and Arenz M 2020 Solvent-Dependent Growth and Stabilization Mechanisms of Surfactant-Free Colloidal Pt Nanoparticles Chem. - A Eur. J. 26 9012-23 
[18] Binninger T, Fabbri E, Patru A, Garganourakis M, Han J, Abbott D F, Sereda O, Kötz R, Menzel A, Nachtegaal M and Schmidt T J 2016 Electrochemical flow-cell setup for in situ X-ray Investigations: I. Cell for SAXS and XAS at synchrotron facilities J. Electrochem. Soc. 163 H906-12

[19] Tillier J, Binninger T, Garganourakis M, Patru A, Fabbri E, Schmidt T J and Sereda O 2016 Electrochemical Flow-Cell Setup for In Situ X-ray Investigations II. Cell for SAXS on a MultiPurpose Laboratory Diffractometer J. Electrochem. Soc. 163 H913-20

[20] Povia M, Herranz J, Binninger T, Nachtegaal M, Diaz A, Kohlbrecher J, Abbott D F, Kim B-J and Schmidt T J 2018 Combining SAXS and XAS To Study the Operando Degradation of Carbon-Supported Pt-Nanoparticle Fuel Cell Catalysts ACS Catal. 8 7000-15

[21] Binninger T, Garganourakis M, Han J, Patru A, Fabbri E, Sereda O, Kötz R, Menzel A and Schmidt T J 2015 Particle-support interferences in small-angle X-ray scattering from supportedcatalyst materials Phys. Rev. Appl. 3

[22] Chaâbane N, Lazzari R, Jupille J, Renaud G and Avellar Soares E 2012 CO-induced scavenging of supported Pt nanoclusters: A GISAXS study J. Phys. Chem. C 116 23362-70

[23] Ruge M, Drnec J, Rahn B, Reikowski F, Harrington D A, Carlà F, Felici R, Stettner J and Magnussen O M 2017 Structural Reorganization of Pt(111) Electrodes by Electrochemical Oxidation and Reduction J. Am. Chem. Soc. 139 4532-9

[24] Khalakhan I, Bogar M, Vorokhta M, Kúš P, Yakovlev Y, Dopita M, Sandbeck D J S, Cherevko S, Matolínová I and Amenitsch H 2020 Evolution of the PtNi Bimetallic Alloy Fuel Cell Catalyst under Simulated Operational Conditions ACS Appl. Mater. Interfaces 12 17602-10

[25] Yarlagadda V, McKinney S E, Keary C L, Thompson L, Zulevi B and Kongkanand A 2017 Preparation of PEMFC Electrodes from Milligram-Amounts of Catalyst Powder J. Electrochem. Soc. 164 F845-9

[26] Kongkanand A and Mathias M F 2016 The Priority and Challenge of High-Power Performance of Low-Platinum Proton-Exchange Membrane Fuel Cells J. Phys. Chem. Lett. 7 1127-37

[27] Wiberg G K H, Mayrhofer K J J and Arenz M 2010 Investigation of the oxygen reduction activity on silver - A rotating disc electrode study Fuel Cells 10 575-81

[28] Quinson J, Inaba M, Neumann S, Swane A A, Bucher J, Simonsen S B, Theil Kuhn L, Kirkensgaard J J K, Jensen K M Ø, Oezaslan M, Kunz S and Arenz M 2018 Investigating Particle Size Effects in Catalysis by Applying a Size-Controlled and Surfactant-Free Synthesis of Colloidal Nanoparticles in Alkaline Ethylene Glycol: Case Study of the Oxygen Reduction Reaction on Pt ACS Catal. 8 6627-35

[29] Speder J, Altmann L, Roefzaad M, Bäumer M, Kirkensgaard J J K K, Mortensen K and Arenz M 2013 Pt based PEMFC catalysts prepared from colloidal particle suspensions - a toolbox for model studies Phys. Chem. Chem. Phys. 153602

[30] Kajiwara K and Hiragi Y 1996 Chapter 6 Structure analysis by small-angle X-ray scattering Anal. Spectrosc. Libr. 7 353-404

[31] Garcia P R A F, Prymak O, Grasmik V, Pappert K, Wlysses W, Otubo L, Epple M and Oliveira C L P 2020 An in situ SAXS investigation of the formation of silver nanoparticles and bimetallic silver-gold nanoparticles in controlled wet-chemical reduction synthesis Nanoscale Adv. 2 22538 
[32] Sandbeck D J S, Inaba M, Quinson J, Bucher J, Zana A, Arenz M, Arenz M and Cherevko S 2020 Particle Size Effect on Platinum Dissolution: Practical Considerations for Fuel Cells ACS Appl. Mater. Interfaces 12 25718-27

[33] Sandbeck D J S, Secher N M, Speck F D, Sørensen J E, Kibsgaard J, Chorkendorff I, Cherevko S and Cherevko S 2020 Particle Size Effect on Platinum Dissolution: Considerations for Accelerated Stability Testing of Fuel Cell Catalysts ACS Catal. 10 6281-90

[34] Yu K, Groom D J, Wang X, Yang Z, Gummalla M, Ball S C, Myers D J and Ferreira P J 2014 Degradation mechanisms of platinum nanoparticle catalysts in proton exchange membrane fuel cells: The role of particle size Chem. Mater. 26 5540-8

[35] Quinson J, Röefzaad M, Deiana D, Hansen T W, Wagner J B, Nesselberger M, Crampton A S, Ridge C J, Schweinberger F F, Heiz U and Arenz M 2018 Electrochemical stability of subnanometer Pt clusters Electrochim. Acta 277 211-7

[36] Cherevko S, Zeradjanin A R, Topalov A A, Kulyk N, Katsounaros I and Mayrhofer K J J 2014 Dissolution of noble metals during oxygen evolution in acidic media ChemCatChem 6 2219-23 